Case Report

\title{
High thrombus burden despite thrombolytic therapy in ST-elevation myocardial infarction in a patient with COVID-19
}

\author{
Gayatri Setia ${ }^{1}$, Jeffrey Tyler ${ }^{2}$, Alan Kwan ${ }^{2}$, Josh Faguet ${ }^{1}$, Shilpa Sharma ${ }^{2}$, Siddharth Singh ${ }^{2}$, Babak Azarbal ${ }^{2, *}$, Rose Tompkins $^{2}$, \\ Dinora Chinchilla ${ }^{3}$ and Sara Ghandehari ${ }^{3}$ \\ ${ }^{1}$ Division of General Internal Medicine, Cedars-Sinai Medical Center, Los Angeles, CA 90048, USA \\ ${ }^{2}$ Department of Cardiology, Cedars-Sinai Medical Center, Los Angeles, CA 90048, USA \\ ${ }^{3}$ Division of Pulmonary and Critical Care Medicine, Cedars-Sinai Medical Center, Los Angeles, CA 90048, USA \\ ${ }^{*}$ Correspondence: Babak.Azarbal@cshs.org (Babak Azarbal)
}

DOI: $10.31083 /$ i.rcm.2020.02.92

This is an open access article under the CC BY-NC 4.0 license (https://creativecommons.org/licenses/by-nc/4.0/).

Consideration of thrombolysis as first-line reperfusion therapy in patients with COVID-19 and STEMI is recommended by ACC/SCAI guidelines. We describe a patient with COVID-19, who presented with ST-elevation myocardial infarction and was treated with thrombolysis and anticoagulation. He was later found to have a significant persistent thrombus burden requiring thrombectomy and stent placement. Invasive hemodynamics on multiple high-dose pressers revealed a high cardiac output state with low systemic vascular resistance, consistent with distributive rather than cardiogenic shock. Our case illustrates that thrombolytic therapy alone may not be adequate in patients with STEMI and COVID-19, as well as the importance of early invasive hemodynamics in management of shock in patient with STEMI and COVID-19 infection.

\section{Keywords}

COVID-19; acute coronary syndrome; ST-elevation myocardial infarction; thrombolysis

\section{Case report}

A 59-year-old man with a history of diabetes, hypertension, and hyperlipidemia presented to the emergency department with substernal chest pain. He also reported a two-week history of cough, pleuritic chest discomfort, and night sweats. On the night prior to admission, about 14-16 hours prior to arrival in the ED, he developed intermittent substernal chest pain, which spontaneously resolved. Chest pain recurred 90 minutes prior to presentation and remained constant and severe, prompting his family to call paramedics. On arrival, he was afebrile, normotensive, bradycardic to 46 beats per minute, tachypneic to 35 breaths per minute, and hypoxemic saturating $75 \%$ on $15 \mathrm{~L}$ of oxygen via non-rebreather. Patient was illappearing and emergently intubated for worsening respiratory distress and hypoxemia.

Initial EKG (Fig. 1A) showed complete heart block (CHB) with a junctional escape rhythm, ST elevations concerning for inferolateral ST elevation myocardial infarction (STEMI). Q waves were evident in the inferior leads, and initial troponin was elevated to $25 \mathrm{ng} / \mathrm{mL}$, suggesting a delayed presentation. Other laboratory tests (Table 1) showed leukocytosis to 12,760 cells $/ \mu \mathrm{L}$ with a left shift and no lymphopenia, increased creatinine level of $1.90 \mathrm{mg} / \mathrm{dL}$, and elevated inflammatory markers including ferritin $(2,634 \mathrm{ng} / \mathrm{dL}), \mathrm{C}$ reactive protein $(259 \mathrm{mg} / \mathrm{L})$, D-dimer $(3.52 \mathrm{ng} / \mathrm{mL} \mathrm{FEU})$, and interleukin-6 (26 pg/mL). Chest X-ray showed patchy bilateral infiltrates concerning for COVID-19 pneumonia. Chest CT angiography excluded pulmonary embolus and aortic dissection, confirmed bilateral infiltrates. Transthoracic echocardiography showed severely reduced left ventricular ejection fraction of $25 \%$ with marked inferolateral wall motion abnormality, mildly depressed right ventricular systolic function, no significant valvular dysfunction, and no pericardial effusion.

Patient received loading doses of aspirin and ticagelor with heparin bolus and drip with a plan for emergent percutaneous coronary intervention (PCI). However, due to a concurrent hemodynamically unstable STEMI occupying the catheterization lab and an anticipated delay of greater than 120 minutes to invasive management, chemical thrombolysis was ultimately 


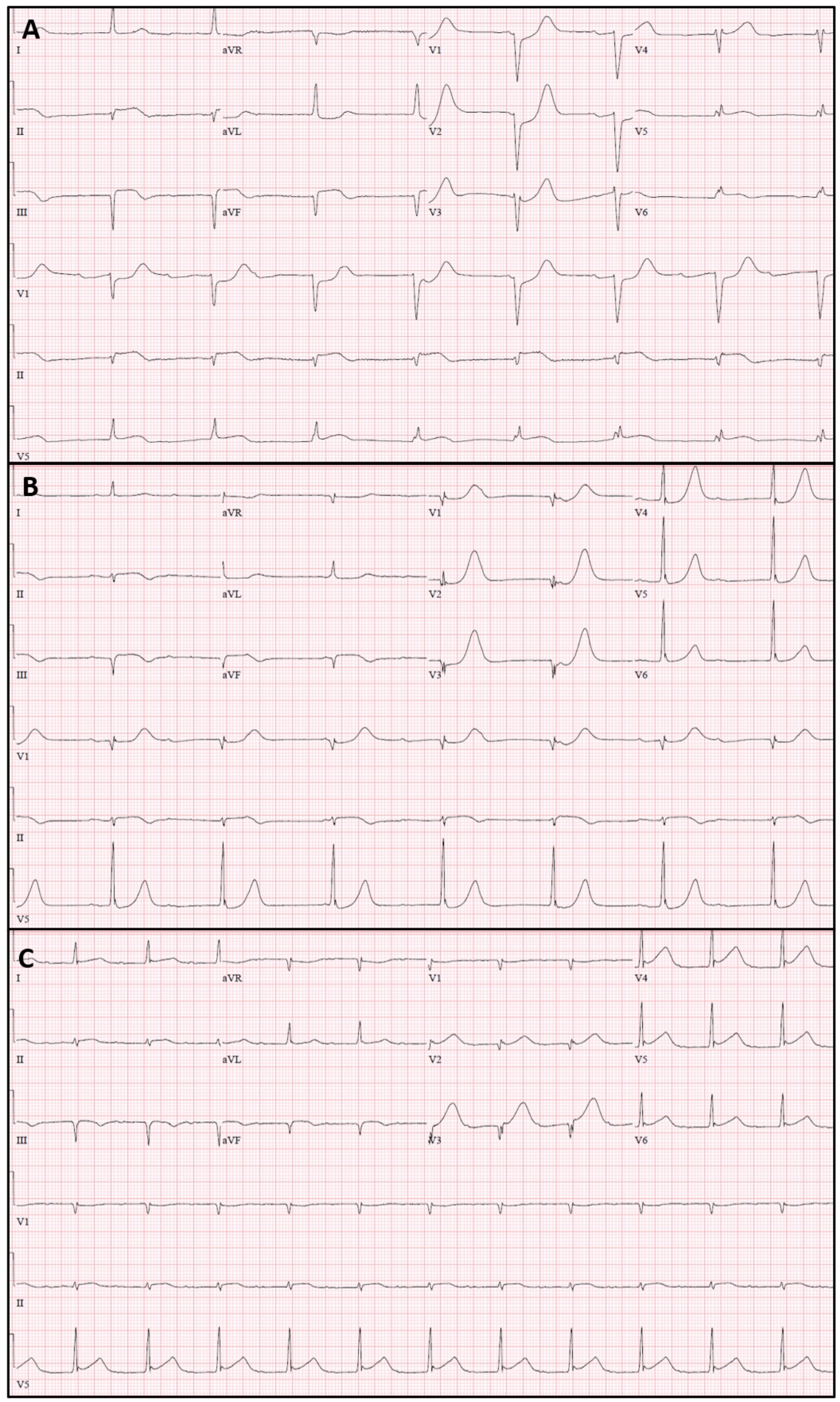

Figure 1. EKG progression throughout hospitalization. A) EKG on admission, B) EKG one hour post-thrombolysis, C) EKG 2 days post-PCI. $\mathrm{PCl}$ : percutaneous coronary intervention. 

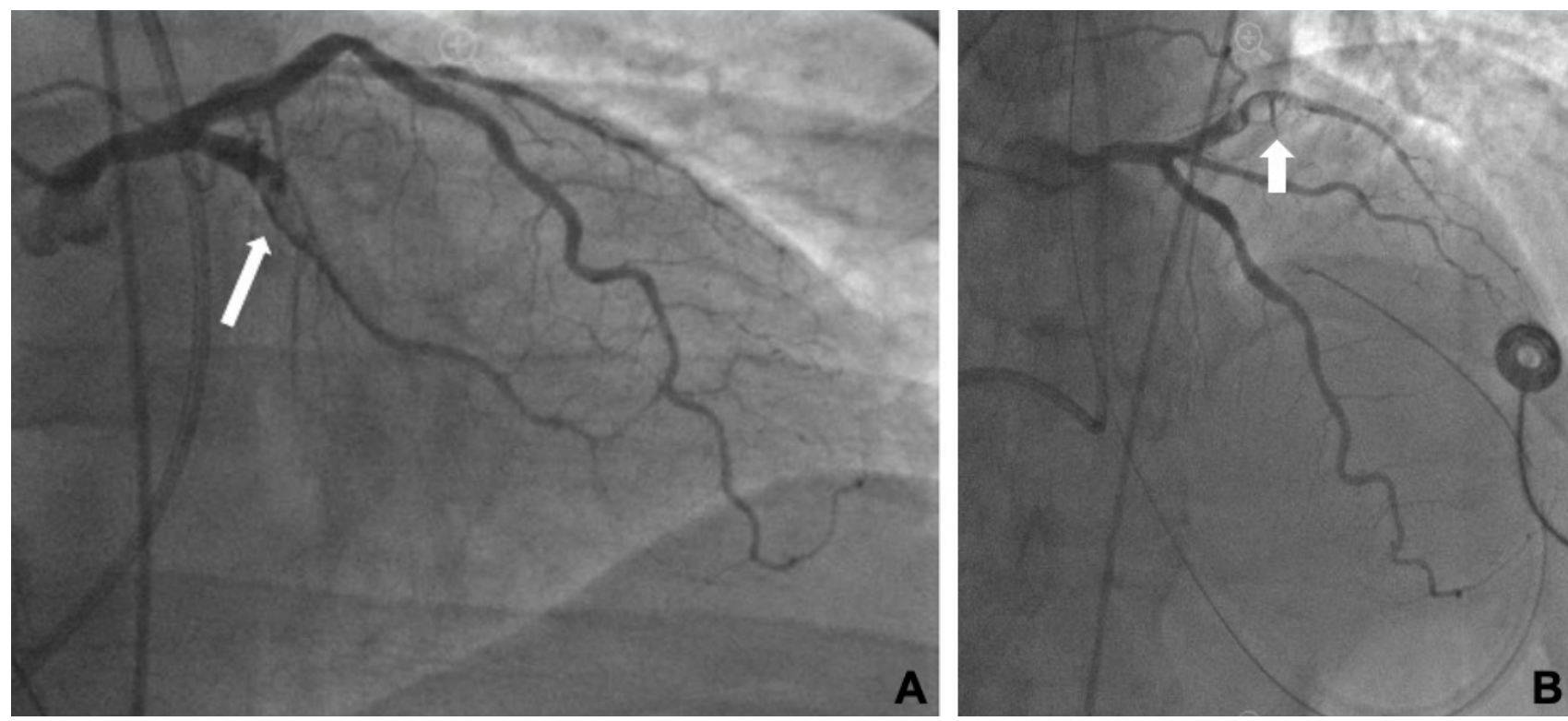

Figure 2. Coronary angiogram of left coronary distributions. A) Caudal and B) cranial projections showing a $90 \%$ thrombotic obstruction in the proximal left circumflex (Arrow) and non-obstructive disease in left anterior descending.

selected as initial reperfusion strategy. Alteplase $15 \mathrm{mg}$ was infused over one minute, followed by $50 \mathrm{mg}$ over 30 minutes, and $35 \mathrm{mg}$ over 60 minutes.

EKG after thrombolysis (Fig. 1B) showed interval improvement of ST elevations. Given the greater than 50\% improvement in ST elevations in the inferior leads and resolution of ST elevations in the lateral leads, thrombolysis was considered successful.

The patient was hypotensive and transferred to the intensive care unit on dopamine, norepinephrine, and vasopressin. Given the EKG improvement after the administration of thrombolysis and significant bilateral consolidations on CT chest, shock was attributed toward most likely mixed cardiogenic and distributive shock from his underlying pulmonary infection. Troponin peaked at $>440 \mathrm{ng} / \mathrm{mL}$. RT-PCR collected on admission returned positive for SARS-CoV-2.

Cardiac catheterization was performed 48 hours after thrombolysis due to persistent mixed shock. Coronary angiography revealed subacute appearing thrombosis in two coronary territories, left circumflex (LCX) and right coronary artery (RCA). A large thrombus was identified in the proximal-to-mid LCX, resulting in $90 \%$ obstruction with TIMI-3 flow (Fig. 2). A clear atherosclerotic lesion could not be identified. The RCA was an ectatic, large, dominant vessel with thrombic occlusion in the distal vessel just after the take-off of the posterior descending artery, which remained patent but was a small (1 mm) vessel with TIMI-2 flow (Fig. 3). There did not appear to be any collateral flow to the distal RCA territory. Mild non- obstructive coronary artery disease was seen in the left anterior descending artery and ramus intermedius. Extensive clot was extracted from the LCX by aspiration thrombectomy. However, due to incomplete removal and persistent occlusion, a single drug-eluting stent was placed with $0 \%$ residual stenosis and maintenance of TIMI-3 blood flow (Fig. 4). The RCA was not intervened upon as the territory was thought to be non-viable due to inferior Q waves noted on EKG.

Prior to PCI, on positive pressure ventilation $\left(5 \mathrm{cmH}_{2} 0\right)$ and vasopressor support, right heart catheterization (RHC) identified the following intracardiac pressures: mean right atrial 9 $\mathrm{mmHg}$, right ventricle $42 / 5 \mathrm{mmHg}$, pulmonary artery $42 / 18$ (26) $\mathrm{mmHg}$, and mean pulmonary artery capillary wedge 17 mmHg. Per thermodilution, the mean cardiac output was 8.2 $\mathrm{L} /$ minute and mean cardiac index was $3.97 \mathrm{~L} / \mathrm{min} / \mathrm{m} 2$, with a systemic vascular resistance (SVR) of 721 dynes, suggesting a high cardiac output state and low SVR on background vasopressor support more consistent with a predominance of distributive versus cardiogenic shock as a primary mechanism for his hypotension.

The patient received empiric broad spectrum antibiotic coverage for possible secondary bacterial pneumonia. Due to fevers and worsening oxygen requirements on hospital day 3 , he received one dose of tociluzimab $400 \mathrm{mg}$ IV offlabel for presumed COVID-19-induced cytokine storm. Given his severe thrombus burden, heparin was continued postcatheterization.

The patient's hemodynamics gradually improved, with atri- 


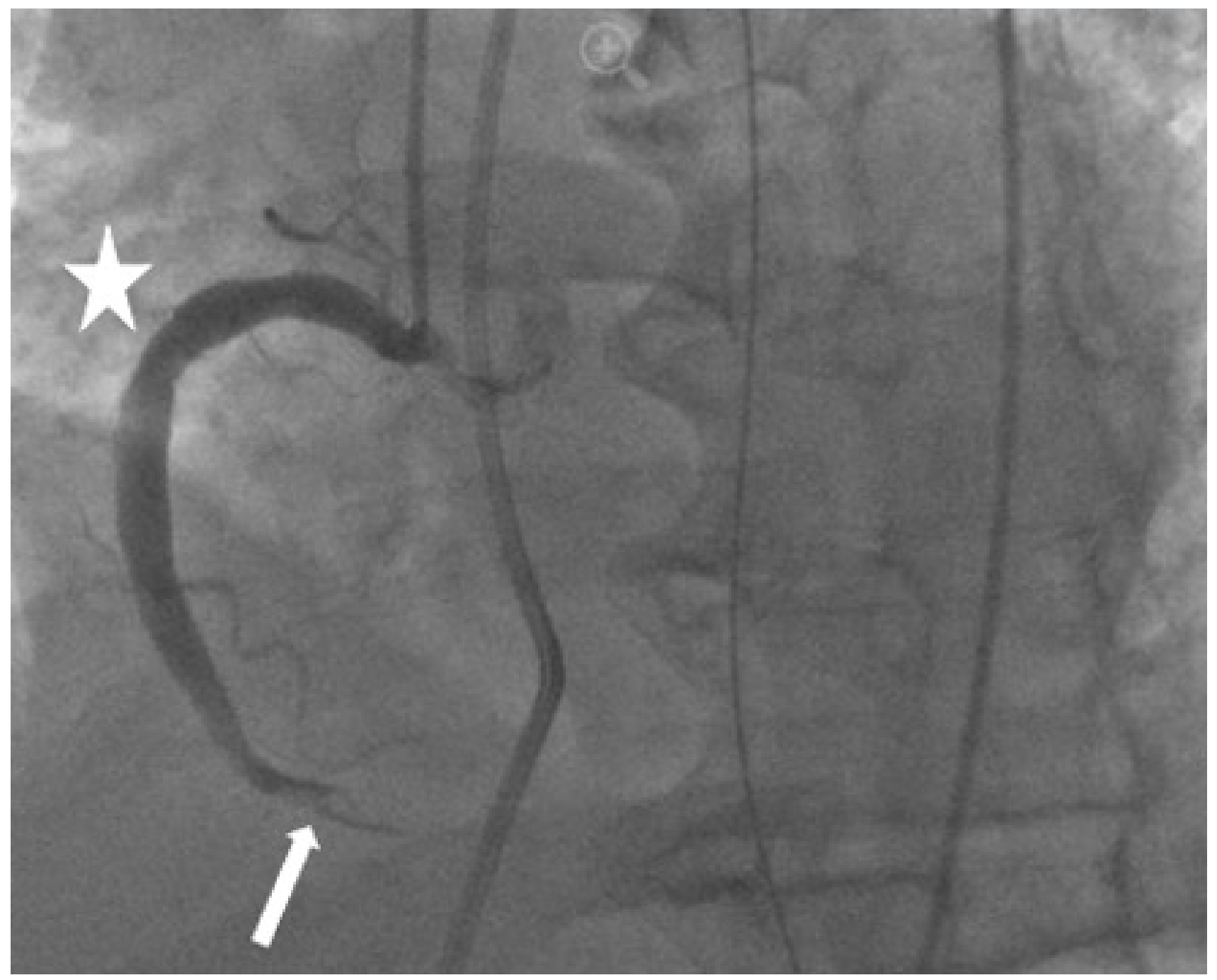

Figure 3. Coronary angiogram of right coronary artery. LAO projection showing an ectatic proximal to mid right coronary artery with mild nonobstructive disease (Star) and a distal vessel with evidence of thrombus prior to the takeoff of the posterior descending artery (Arrow). LAO: left anterior oblique.

oventricular node recovery noted by hospital day 4 (Fig. 1C) and successful weaning off of all vasoactive drips by day 7 . Oxygen requirements improved, and he was extubated on day 13. The patient was discharged on hospital day 19 on apixaban and ticagrelor and, on follow-up phone conversations, continues to improve symptomatically.

\section{Discussion}

Currently, pathophysiology of the COVID-19 virus is incompletely understood thus creating clinical management challenges. Hypercoagulability and increased rate of arterial thrombosis in multiple vascular beds, including coronary thrombosis causing STEMI, has been observed in patients with COVID-19 (Tan et al., 2020; Tang et al., 2020; Zhang et al., 2020). Proposed mechanisms of hypercoagulability and arterial thrombosis include development of antiphospholipid an- tibodies, complement-mediated endothelial injury, and plaque destabilization due to systemic inflammation (Campbell and Kahwash, 2020; Xiong et al., 2020; Zhang et al., 2020). Consideration of thrombolysis for first-line treatment of STEMI in order to decrease catheterization lab exposure has been suggested for COVID-19 positive patients in ACC/SCAI guidelines (Welt et al., 2020). A case series of STEMI in COVID-19 patients by Bangalore et al. (2020) observed inferior outcomes and a $100 \%$ prevalence of elevated D-dimer relative to STEMI patients with COVID-19 (Bangalore et al., 2020). Importantly, most of the patients in this series were managed with emergent cardiac catheterization and not thrombolysis, but notably still with poor outcomes. Bangalore et al. (2020) also found that 10 out of their 18 COVID-19-positive patients with ST elevations on EKG ultimately had noncoronary myocardial injury, 


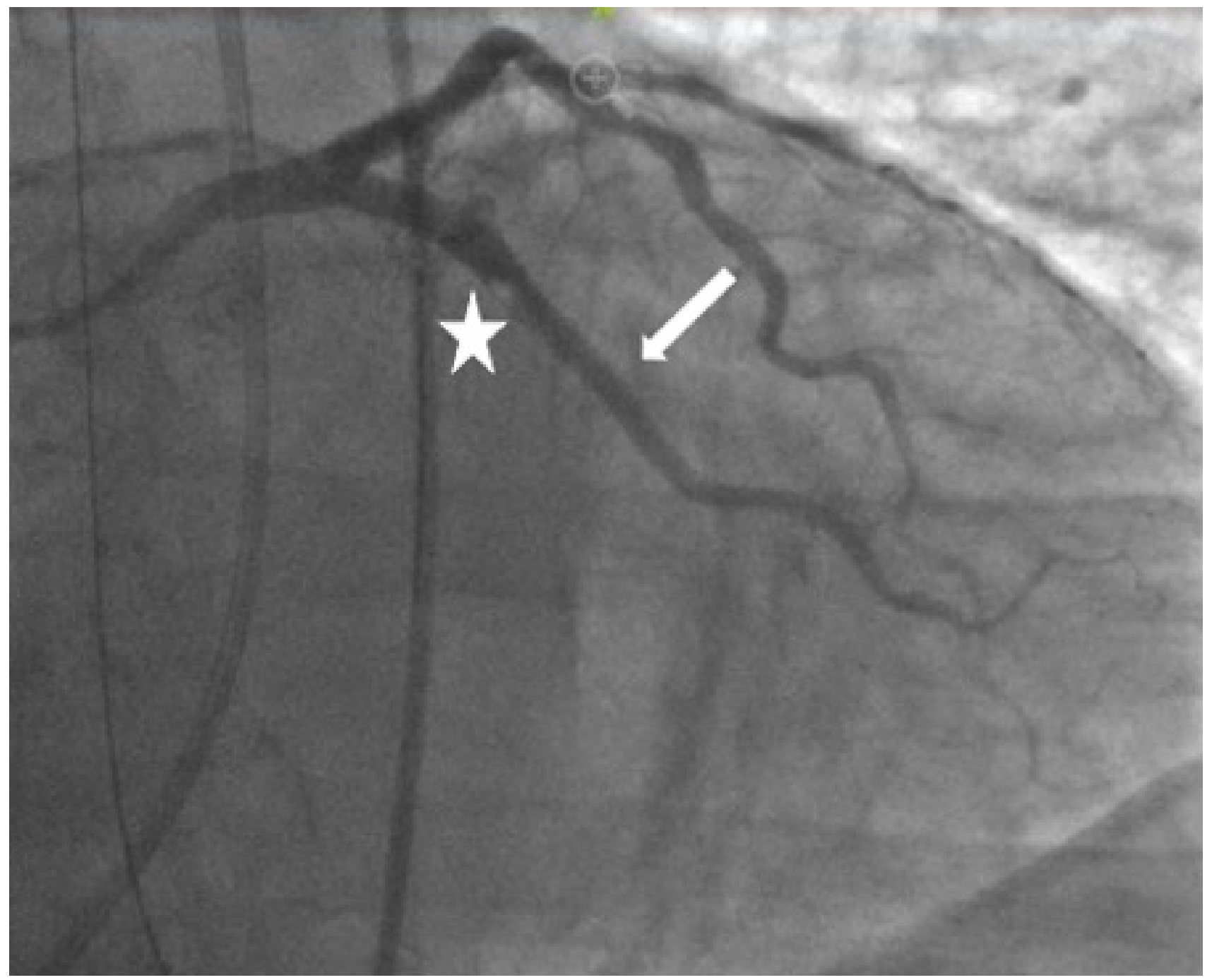

Figure 4. Coronary angiogram post- $\mathrm{PCl}$ of the left circumflex artery. Successful $\mathrm{PCl}$ of the left circumflex with a drug-eluting stent extending from the proximal left circumflex to the second obtuse marginal branch (Arrow). There is a nonobstructive lesion in the mid left circumflex coronary artery that was significantly reduced in size but could not be completely obliterated following $\mathrm{PCl}$ (Star). PCl: percutaneous coronary intervention.

which is an important consideration to make when deciding whether to give thrombolytics to someone and exposing them to a higher risk of bleeding (Bangalore et al., 2020). It should be noted that, at the time of presentation, it was not known that the patient had a COVID-19 infection. At the time, due to the typical chest pain pattern and the EKG pattern, the pretest probability of coronary obstruction was high enough for this patient to proceed with thrombolysis. While our patient had risk factors for coronary artery disease, we believe that his presentation of heavy thrombus burden in two coronary distributions despite lytic therapy and anticoagulation may be out of proportion to his risk. We believe his coronary occlusions were due to simultaneous thrombotic occlusions triggered by the hypercoagulable state associated with COVID-19, indicating fur- ther evidence of a different pathophysiology causing STEMI in patients with COVID-19. Given the rapid practice changes related to thrombolytic therapy in the midst of the COVID-19 pandemic, we believe that this case demonstrates a need for increased caution in exclusively depending on thrombolysis in treating COVID-19 patients with STEMI, as our patient likely benefited from eventual percutaneous coronary intervention as well as continuation of anticoagulation therapy.

Additionally, RHC and invasive hemodynamics proved important in guiding management of this patient in his mixed shock state, as patients with concurrent COVID-19 infection and STEMI can have multiple etiologies for shock and respiratory failure. Despite this patient's large STEMI and severely reduced left ventricular systolic function, the invasive hemody- 
Table 1 . Initial laboratory values on admission

\begin{tabular}{lcc}
\hline Laboratory Values & Result & Reference Range \\
\hline White-cell Count & $12,760 \mathrm{cells} / \mathrm{mL}$ & $4,000-11,000 \mathrm{cells} / \mathrm{mL}$ \\
- Neutrophils \% & $74 \%$ & \\
- Bands \% & $8 \%$ & \\
- Lymphocytes \% & $11 \%$ & \\
Troponin & $25.33 \mathrm{ng} / \mathrm{mL}$ & $<0.04 \mathrm{ng} / \mathrm{mL}$ \\
Creatinine & $1.90 \mathrm{mg} / \mathrm{dL}$ & $0.72-1.25 \mathrm{mg} / \mathrm{dL}$ \\
AST & $220 \mathrm{U} / \mathrm{L}$ & $5-34 \mathrm{U} / \mathrm{L}$ \\
ALT & $65 \mathrm{U} / \mathrm{L}$ & $0-55 \mathrm{U} / \mathrm{L}$ \\
Lactate & $4.5 \mathrm{mmol} / \mathrm{L}$ & $0.5-2.2 \mathrm{mmol} / \mathrm{L}$ \\
Lactate Dehydrogenase & $1,246 \mathrm{U} / \mathrm{L}$ & $125-220 \mathrm{U} / \mathrm{L}$ \\
Ferritin & $2,634 \mathrm{ng} / \mathrm{mL}$ & $21-275 \mathrm{ng} / \mathrm{mL}$ \\
C-Reactive Protein & $259.2 \mathrm{mg} / \mathrm{L}$ & $<5 \mathrm{mg} / \mathrm{L}$ \\
D-Dimer & $3.52 \mathrm{ng} / \mathrm{mL} \mathrm{FEU}$ & $0.50-0.60 \mathrm{ng} / \mathrm{mL} \mathrm{FEU}$ \\
Procalcitonin & $0.43 \mathrm{ng} / \mathrm{mL}$ & $<0.08 \mathrm{ng} / \mathrm{mL}$
\end{tabular}

namic values obtained supported a predominance of distributive shock, and use of temporary mechanical circulatory support or intra-aortic balloon pump counterpulsation were unlikely to be of benefit.

In conclusion, this case demonstrates a multi-territory STEMI in a patient with COVID-19, with extreme clot burden despite thrombolysis and greater than 48 hours of heparin therapy. Thrombolytic therapy alone in COVID-19-positive patients with STEMI, as proposed by national guidelines, should be used with caution. Additionally, in the setting of mixed shock, hemodynamic and volume management guided by RHC could be an important tool in caring for these patients.

\section{Authors' contributions}

Gayatri Setia MD - Data curation, writing - original draft, writing - review \& editing

Jeffrey Tyler MD - Data curation, writing - review \& editing, Supervision, Visualization

Alan Kwan MD - Writing - review \& editing, Data curation, Visualization

Josh Faguet MD - Writing - review \& editing

Shilpa Sharma MD -Writing - review \& editing

Siddharth Singh MD - Writing - review \& editing

Babak Azarbal MD - Writing - review \& editing, Supervision, Conceptualization

Rose Tompkins MD - Writing - review \& editing, Conceptualization

Dinora Chinchilla MD - Writing - review \& editing
Sara Ghandehari MD - Writing - review \& editing, Supervision, Conceptualization

\section{Ethics approval and consent to participate}

As this is a case report intended for medical and educational purposes, it does not meet regulation criteria necessitating IRB approval, and therefore no IRB approval was obtained. Consent for publication was obtained by the patient and the patient's family.

\section{Acknowledgments}

The authors declare no funding, no contribution from other persons.

\section{Conflict of interest}

The authors declare no conflicts of interests.

Submitted: May 15, 2020

Revised: June 17, 2020

Accepted: June 22, 2020

Published: June 30, 2020

\section{References}

Bangalore, S., Sharma, A., Slotwiner, A., Yatskar, L., Harari, R., Shah, B., Ibrahim, H., Friedman, G. H., Thompson, C., Alviar, C. L., Chadow, H. L., Fishman, G. I., Reynolds, H. R., Keller, N. and Hochman, J. S. (2020) ST-Segment elevation in patients with Covid-19 - A case series. The New England Journal of Medicine 382, 2478-2480.

Campbell, C. M. and Kahwash, R. (2020) Will complement inhibition be the new target in treating COVID-19-related systemic thrombosis? Circulation 141, 1739-1741. 
Tan, C. W., Low, J. G. H., Wong, W. H., Chua, Y. Y., Goh, S. L. and Ng, H. J. (2020) Critically ill COVID-19 infected patients exhibit increased clot waveform analysis parameters consistent with hypercoagulability. American Journal of Hematology 95, E156E158.

Tang, N., Bai, H., Chen, X., Gong, J., Li, D. and Sun, Z. (2020) Anticoagulant treatment is associated with decreased mortality in severe coronavirus disease 2019 patients with coagulopathy. Journal of Thrombosis and Haemostasis 18, 1094-1099.

Welt, F. G. P., Shah, P. B., Aronow, H. D., Bortnick, A. E., Henry, T. D., Sherwood, M. W., Young, M. N., Davidson, L. J., Kadavath, S., Mahmud, E., Kirtane, A. J., American College of Cardiology's Interventional Council and the Society for Cardiovascular Angiography and Interventions (2020) Catheterization laboratory consid- erations during the Coronavirus (COVID-19) pandemic: From the ACC's Interventional Council and SCAI. Journal of the American College of Cardiology 75, 2372-2375.

Xiong, T.-Y., Redwood, S., Prendergast, B. and Chen, M. (2020) Coronaviruses and the cardiovascular system: acute and long-term implications. European Heart Journal 41, 1798-1800.

Zhang, Y., Xiao, M., Zhang, S., Xia, P., Cao, W., Jiang, W., Chen, H., Ding, X., Zhao, H., Zhang, H., Wang, C., Zhao, J., Sun, X., Tian, R., Wu, W., Wu, D., Ma, J., Chen, Y., Zhang, D., Xie, J., Yan, X., Zhou, X., Liu, Z., Wang, J., Du, B., Qin, Y., Gao, P., Qin, X., Xu, Y., Zhang, W., Li, T., Zhang, F., Zhao, Y., Li, Y. and Zhang, S. (2020) Coagulopathy and antiphospholipid antibodies in patients with Covid-19. The New England Journal of Medicine 382, e38e38. 